\title{
Remote Sharing Network (RSN): A Collaborative Platform
}

\author{
Raksha N Bysani \\ Department of Computer \\ Science \& Engineering \\ BMS College of Engineering \\ Bengaluru, India
}

\author{
Satyaprakash K N \\ Project Guide \\ ESI Software (I) Pvt. Ltd. \\ Bengaluru, India
}

\author{
Umadevi V \\ Project Guide, Department of \\ Computer Science \& Engg. \\ BMS College of Engineering \\ Bengaluru, India
}

\begin{abstract}
Collaborative development platform has become an important business strategy. A collaborative platform may facilitate the development and deployment of a collaborative environment. New or existing data can be communicated via the collaborative platform on various local environments to create a collaborative environment among multiple participants. The smartphone is a tool for both communication and productivity. As a result, it is the ideal device for collaboration. Through the collaborative technology different project stakeholders can discuss changes and requirements. The forum lets designers, systems engineers, project managers and other team members discuss projects on $3 \mathrm{D}$ models virtually as if they were having a round table discussion. This helps teams communicate more efficiently, saving the business time while increasing efficiency. The RSN Software solution is a robust communication tool that lets product development teams track projects in a transparent way. The key benefit of RSN Software is the ability to effectively exchange engineering data in the workflow processes for quick decision-making. It lets users check whether designs have adhered to the acceptable norms and ensures that site staff uses the latest designs, making quality assurance a much easier task and reducing the cost of errors.
\end{abstract}

\section{Keywords}

Collaborative Platform, NoSQL database, Push Notification, Cross Platform

\section{INTRODUCTION}

Engineers deal with drawings in 2D and 3D data while engineering a product. In addition to this, the design involves a lot of mathematical calculations and the response of the product is studied by plotting different graphs. The product design also involves team members working from different geographical locations to share the above data and engineer the product. Today there is no platform which can aid the team in exchanging product information during the product development. The Engineers need to attend formal meetings to take any necessary decisions and it becomes very difficult to cooperate when the people involved in the project managers, consultants, technicians, etc are from different geographical locations. Collaboration among them is required at every stage of project management - which includes project conception, planning, budget preparation and implementation. Hence the availability of a collaborative platform will (1) Reduce the time (2) Reduce cost required for project management and product development (3) Uniting all partners involved in the product development life cycle. What we need is a platform for the online communication of all the project players and the process controlled provision and storage of project documents in the cloud or for the mobile collection of actual data at the engineering site.

\section{RELATED RESEARCH}

\subsection{Need of a Collaborative Platform}

A collaborative platform is a type of business software that adds broad social networking capabilities to work processes. The goal of a collaboration platform application is to foster innovation by incorporating knowledge management into business processes so employees can share information and solve business problems more efficiently. It helps give the team Confluence, as we do not require the need of emails and meetings and hence reducing the problems. In [1] as stated, a Collaborative Platform is a technology designed to facilitate communication, to cooperate, coordinate, solve problems, or to deal with negotiations of work groups. This elaborates the characteristics of the Decision Support System (DSS) against the Collaborative Platform.

\subsection{Existing Collaborative Platforms}

There are several collaborative platforms that exist. SyTron is one which supports e-learning system by providing a virtual classroom facilitating effective communication between the teachers and students via voice and text based information exchange [2]. Practo is a platform that provides online medical practice for doctors and facilitates the doctor-patient relationship [3]. ITWOcx is another example of a collaborative platform that facilitates exchange of various forms of data being dealt with by construction and infrastructure projects [4]. The existing platforms like these suggest the need of a platform like RSN solution to help exchange engineering data, i.e., 3D models, 2D graphs etc.

\subsection{Software that helps Facebook scale}

This research begins with examining the various tools and technologies used to build a social communication platform like Facebook. As mentioned in website citation [5], Facebook was built from scratch using tools like PHP, Linux and MySQL, but has now modified these to scale it to accommodate the website that we see today. For example, Facebook has optimized Linux to suit its requirements. In addition to these traditional software, Facebook has modified the operation by using custom-written software that are mentioned below.

Haystack is a scalable software that stores the massive amount of photos, videos, messages, etc. Scribe is a userlogging system that is highly scalable enough to handle the current population and more. A few other softwares that Facebook uses that are relevant for building a collaborative platform are: 


\subsubsection{Cassandra}

Cassandra is an open source distributed database management system (running on NoSQL) which is robust and can handle data across many servers. It is described as having no "single point of failure". Facebook uses this software for its Inbox search.

\subsubsection{Scribe}

Scribe is defined as a "flexible logging system that Facebook uses for a multitude of purposes internally". It is highly scalable and can handle large number of logs at a time. It was built to accommodate the exponential growth of the use of Facebook as an application.

\subsubsection{Hadoop and Hive}

Hadoop is a tool to perform complex calculations on large amounts of data, otherwise known as big data. We can imagine the volume of data generated by Facebook, all this data is analyzed to derive current trends and patterns to help in introduction of new trendy features. This task is facilitated by open source tools such as Hadoop and Hive. These tools have gained popularity over the years and are increasingly being used by big corporations.

\subsubsection{Thrift}

Facebook cannot provide all the features by using one particular language, i.e., there is no language that is powerful enough. So it uses multiple languages like PHP for the frontend, Erlang for Chat, it also uses Java and $\mathrm{C}++$ in several places. The website [5] defines Thrift as a cross-language framework that integrates all the above different languages together. This allows these languages to work with each other and hence make the necessary services available.

\section{RSN SOLUTION AND ITS IMPLEMENTATION}

The RSN Solution has 3 modules:

\subsection{Database}

RSN solution provides integrated project document control, collaboration, contract administration, defects and quality management and dashboard capability via the cloud and mobile devices. The RSN solution requires various software and a scalable as well as a consistent database to help build the collaborative platform. In [6] it is stated that, NOSQL databases are different from traditional databases in that they do not use SQL query language, no join operations and BASE is supported instead of ACID (which is the core concept of relational databases). In [7] it says how NoSQL database systems are today an effective solution to manage large data sets distributed over many servers. An important aspect of interest in NoSQL systems is their support for next generation web applications, for which relational DBMSs are not well suited. These are simple applications for which (i) data have a structure that does not fit well in the rigid structure of relational tables, (ii) access to data is based on simple readwrite operations, (iii) scalability and performance are important quality requirements, and a certain level of consistency is also desirable [8]. More than fifty NoSQL systems exist [9], each with different characteristics.
Table 1. Categorization and Comparison of Different NoSQL Databases

\begin{tabular}{|c|c|c|c|c|}
\cline { 2 - 5 } \multicolumn{1}{c|}{} & Performance & Ncalability & Flexibility & Complexity \\
\hline $\begin{array}{c}\text { Key value } \\
\text { stores } \\
\text { (Redis, } \\
\text { Scalaris })\end{array}$ & High & High & High & None \\
\hline $\begin{array}{c}\text { Column } \\
\text { stores } \\
\text { (Apache } \\
\text { CouchDB })\end{array}$ & High & High & Moderate & Low \\
\hline $\begin{array}{c}\text { Document } \\
\text { stores } \\
(\text { CouchDB, } \\
\text { MongoDB })\end{array}$ & High & High & High & Low \\
\hline $\begin{array}{c}\text { Graph } \\
\text { databases } \\
(\text { Neo4j })\end{array}$ & Variable & Variable & High & High \\
\hline
\end{tabular}

They can be classified into a few main categories [8] like keyvalue stores, document stores, and extensible record stores. There are various NoSQL databases like Mongo DB, CouchDB, Cassandra etc, and in [10]-[11][Table 1] it states the various pros and cons of the different NoSQL databases. Cassandra is an open source distributed database management system which is robust and can handle data across many servers. It is written in java and when you need to store data so huge that it doesn't fit on server, but still want a friendly familiar interface to it. CouchDB is a classic document and BigTable stores. Its main point is the Database consistency, the ease of use and written in Erlang. CouchDB can be used for accumulating, occasionally changing data, on which predefined queries are to be run and places where versioning is important. Mongo DB is one of the popular NoSQL database and is written in $\mathrm{C}++$. It retains some of the friendly properties of SQL. And can be used if there is a need of dynamic queries, preferably to define indexes, not map/reduce functions.

\subsection{Push Notifications}

A key requirement of the RSN solution is to keep the mobile users up-to-date as events occur in some data. An event can be the receiving of new data or changes made to existing data.

One way of providing timely updates is the use of push notification technology. In article [12], the paper introduces the various design issues for push notifications and reveals methods of implementing push notifications for Android and iOS using GCM (Google Cloud Messaging) and APNS (Apple Push Notification Service) respectively. Table [2] provides the different implementations of the two services. 
Table 2. Features of GCM and APNS

\begin{tabular}{|c|c|c|c|}
\hline & & $\begin{array}{c}\text { GCM } \\
\text { (Google } \\
\text { Cloud } \\
\text { Messaging) }\end{array}$ & $\begin{array}{c}\text { APNS (Apple } \\
\text { Push } \\
\text { Notification } \\
\text { Service) }\end{array}$ \\
\hline \multirow{2}{*}{$\begin{array}{l}\text { Programming } \\
\text { model }\end{array}$} & $\begin{array}{c}\text { Notification } \\
\text { paradigm }\end{array}$ & $\begin{array}{l}\text { Point-to- } \\
\text { point }\end{array}$ & Point-to-point \\
\hline & $\begin{array}{c}\text { Client } \\
\text { Platform }\end{array}$ & Android 2.2+ & iOS \\
\hline Reliability & $\begin{array}{l}\text { Delivery } \\
\text { guarantees }\end{array}$ & $\begin{array}{l}\text { Fire-and- } \\
\text { forget }\end{array}$ & $\begin{array}{l}\text { Fire-and- } \\
\text { forget }\end{array}$ \\
\hline Constraints & $\begin{array}{c}\text { Notification } \\
\text { Payload } \\
\text { Size }\end{array}$ & 4 Kbytes & 256 bytes \\
\hline
\end{tabular}

The RSN application has a push notification service integrated in it. Push notifications notify the user when an event takes place in the application. The user can respond to the notification at any later time without disrupting the task he is currently doing. Notifications can be of two types - Push and Pull notifications. A comparative and explanatory study amongst the two is provided in article [16]. Paper [13] presents a component model for push notifications that enables to compare different implementation methods of push notifications. The website cited in [14] provides a detailed step-by-step explanation for an android developer to implement Push services using GCM. GCM or Google Cloud Messaging allows servers to send messages (ranging from a few bytes to as large as $4 \mathrm{~KB}$ of payload size) to Android devices and vice versa, upstream data from the device back to the server. The website cited in [15] explains the APNS which is a robust tool for implementing push services on iOS devices. The messages are sent to a server that is maintained by Apple and further forwarded to the receiving device. Each device registers itself with the server and receives messages persistently. When the app is not open on the device, the user is notified of the changes and can be act upon it at a later time.

\subsection{Visualization and Cross-platform tools}

There are three types of mobile applications we can create Native, Web and Hybrid. A study of these from article [18] tells that native apps are installed through the application store particularly for a single platform. They are created for a single platform with the help of SDKs and frameworks. Web apps are not real apps but rather websites that give the native look and feel of a native app and are run on a browser. Mobile web applications are developed as a single web site for all use on all devices with the help of frameworks. Hybrid apps are a combination of both web apps and native apps that take advantage of device features. Article [19] focuses on the need of a cross platform framework for mobile apps. All mobile platforms (Android, Windows, iOS) differ from each other, so the software developers have to develop an app for each platform if they want to reach a large audience which will be cumbersome. Cross platform application development with the help of various tools can help us overcome this disadvantage to a great extent by designing and coding across different platform. The RSN solution is to be developed using a cross platform framework to facilitate access on different devices.
The various tools that could be used for the development of such an app are Phonegap, Titanium Mobile, Rhodes, Kivy, jQuery Mobile, Sencha Touch, Qt etc. Table 3 and Table 4 shows a comparison of some important cross platform tools we came across [19] - [22].

Marmalade SDK allows us to write code and compile on Xcode (Mac OSx) or Microsoft Visual Studio (Windows). Applications are written in C++. It supports OpenGL ES 1.0 to 3.0. Marmalade Hub is the user-friendly GUI tool [24].

Xamarin helps us to write our apps in C\# and deploy the code on iOS, Android, Windows platforms. Xamarin Studio is the IDE [25].

Table 3. Comparison Of Cross Platform Tools

\begin{tabular}{|c|c|c|c|}
\hline Tools & $\begin{array}{c}\text { Platforms } \\
\text { supported }\end{array}$ & License & $\begin{array}{c}\text { Open } \\
\text { source }\end{array}$ \\
\hline PhoneGap & $\begin{array}{c}\text { Android, iOS, } \\
\text { Windows, } \\
\text { BlackBerry }\end{array}$ & Apache 2.0 & Yes \\
\hline Titanium & $\begin{array}{c}\text { Android, iOS, } \\
\text { BlackBerry }\end{array}$ & $\begin{array}{c}\text { Apache } \\
\text { public } \\
\text { license v2 }\end{array}$ & $\begin{array}{c}\text { Yes (with } \\
\text { limited } \\
\text { features) }\end{array}$ \\
\hline Marmalade & $\begin{array}{c}\text { iOS, Android, } \\
\text { Windows, } \\
\text { Blackberry } \\
\text { and Mac OSx }\end{array}$ & Proprietary & Yes \\
\hline \multirow{2}{*}{ Qt } & $\begin{array}{c}\text { Android, iOS, } \\
\text { Windows, } \\
\text { BlackBerry, } \\
\text { Mac OSx }\end{array}$ & - & $\begin{array}{c}\text { Yes (with } \\
\text { limited } \\
\text { features) }\end{array}$ \\
\hline & $\begin{array}{c}\text { Android, iOS, } \\
\text { Mindows, }\end{array}$ & $\begin{array}{c}\text { GNU } \\
\text { General } \\
\text { Public } \\
\text { License }\end{array}$ & Yes \\
\hline
\end{tabular}

Table 4. Comparison Of Cross Platform Tools

\begin{tabular}{|c|c|c|c|}
\hline Tools & $\begin{array}{c}\text { Development } \\
\text { Environment }\end{array}$ & Type & $\begin{array}{c}\text { Type of } \\
\text { app }\end{array}$ \\
\hline PhoneGap & $\begin{array}{c}\text { Native IDE of } \\
\text { the mobile } \\
\text { platform }\end{array}$ & Framework & Hybrid \\
\hline Titanium & $\begin{array}{c}\text { Titanium } \\
\text { Studio } \\
\text { (Eclipse-based) }\end{array}$ & Platform & Native \\
\hline Xamarin & $\begin{array}{c}\text { Preferred IDE } \\
\text { (Visual Studio } \\
\text { or Xcode) }\end{array}$ & $\begin{array}{c}\text { Game } \\
\text { Engine }\end{array}$ & Hybrid \\
\hline Studio & Qt Creator & Framework & $\begin{array}{c}\text { Native/ } \\
\text { Hybrid }\end{array}$ \\
\hline
\end{tabular}

Qt is a cross-platform application and UI framework for developers using C++. Qt Creator is supporting Qt IDE. Qt Designer helps to create the UI files with the help of simple drag and drop functions [26].

Visualization deals with the way images, videos, audios and other forms of data appear on the device. Mobile devices are characterized by a small display size, low processing power and less memory capacity. The last two points make it difficult to render 3D graphics [23]. 


\section{DESIGN}

RSN Solution is a collaborative platform for Engineers. RSN solution software is a fully integrated software platform spanning the entire lifecycle of engineering projects. The prototype as shown in Figure 1 will involve the following components: a server deployed in the cloud to facilitate exchange and storage of information. It is proposed to use a NoSQL DB like MongoDB, for managing the data. Dedicated apps are required to be written on Window/MAC/iOS/Android platforms along with push notification services. The user management module and the content management module manage the users and the content respectively.

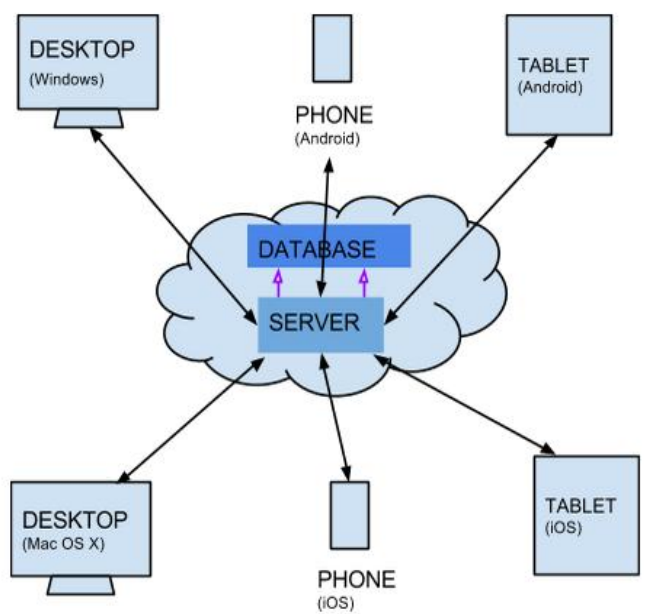

Figure 1. Overview of RSN Solution

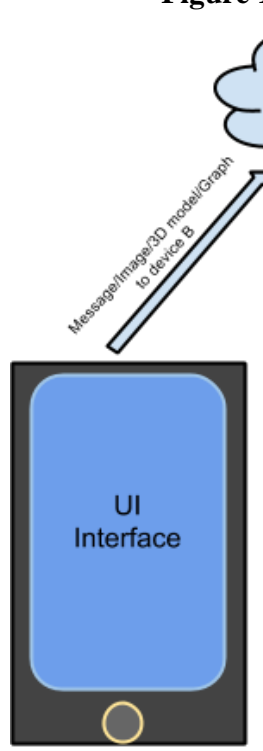

Device A

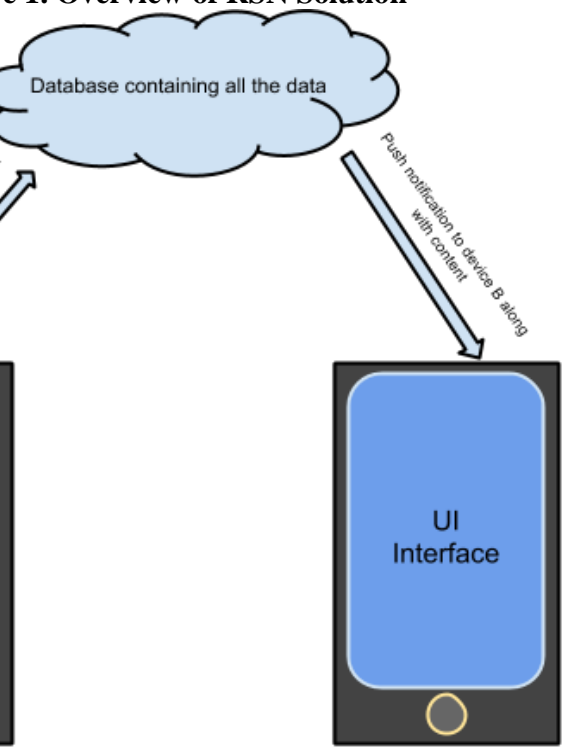

Device B
Figure 2. Implementation of RSN solution

Figure 2 shows the implementation of the RSN solution. The application is developed using Qt which is a cross platform framework. The application is deployed on iOS platform. The data is created on the Desktop whereas data can be viewed and modified on the mobile devices. The data created on the desktop is stored in the MongoDB database in the server deployed on the cloud. Spring Services provides a Mongo
Template and a GridFS Template for dealing with Mongo. MongoDB stores data in JSON format. Push Notifications are implemented by registering to the APNS for iOS. Engineers communicate with data in various forms: Messages, Images, Videos, 2D Graphs, and 3D models. Users can log onto the app using a unique login ID and password (hence registering themselves on the application server). They can communicate with other engineers and collaborate information like designs required for product development, ideas for implementation, etc.

\section{CONCLUSION}

The use of RSN Solution in the workplace creates a collaborative working environment. The solution supports people in both their individual and cooperative work thus giving birth to a new class of professionals, e-professionals, who can work together irrespective of their geographical location.

We provided detailed information about the features and the need of RSN solution. We presented the three different modules that are integrated to create the RSN solution. Each module elaborates the different technologies that have been explored with respect to the requirements of the solution. A framework for RSN Solution has been developed using Qt and MongoDB for frontend and backend respectively.

\section{ACKNOWLEDGMENTS}

We are grateful to BMS College of Engineering for having provided us with the facilities needed for the successful completion of this work. We would also like to thank the company, ESI Software Pvt. Ltd, Bengaluru, for giving us the opportunity to work on this project. We are grateful to Mahesh Hariharan Iyer for his guidance. We would like to thank Nikita Ravindran and Sheena H Jain for being a part of this research. Finally, we would like to thank our parents and friends for their constant support and encouragement. The work reported in this paper is supported by the college through the TECHNICAL EDUCATION QUALITY IMPROVEMENT PROGRAMME [TEQIP-II] of the MHRD, Government of India.

\section{DECLERATION}

This article/typescript has not been submitted for publication elsewhere.

\section{REFERENCES}

[1] M. Bîzoi, A.M. Suduc,F.G. Filip "Using Collaborative Platforms for Decision Support" Faculty of Electrical Engineering, University of Valahia, Tagoviste, Romania. Romanian Academy, Bucharest, Romania, 2009, pp. 349-352.

[2] R.Chellali, C. Dumas, G. Subleau, Nicolas Mollet, "SyTroN: a virtual classroom for collaborative and distant e-learning system by tele operating real devices", International Journal of Computer Games Technology Volume 2009, January 2009 Article No. 4

[3] "PRACTO RAY, medical practice management platform". [Online] Available: https://www.practo.com/ray [Accessed Jan 16th, 2015]

[4] "ITWOCX (Collaboration Exchange)". [Online] Available: https://aucdn01.pcstatic.net/files/whats_new_itwocx.pdf [Accessed Jan 15th, 2015] 
[5] "Exploring the software behind Facebook, the world's largest site" Posted in Tech blog on June 18th, 2010 by Pingdom.Available:http://royal.pingdom.com/2010/06/1 8/the-software-behind-facebook/ [Accessed Jan 15th, 2015]

[6] NaseerGainee, DrRuchiraBhargava, "NoSQL for interactive applications", International journal of Allied Practice, Research and Review, Vol 1, No. 1, pp. 6-7.

[7] Francesca Bugiotti, Luca Cabibbo, Paolo Atzeni, Riccardo Torlone, "Database Design for NoSQL Systems", [Technical Report] Inria\&Universite Paris-Sud, Universita Roma Tre, June 2014.

[8] Rick Cattell. "Scalable SQL and NoSQL data stores", [Technical Report] SIGMOD Record, Vol 39, No. 4, 2010, pp. 12-27.

[9] Michael Stonebraker. "Stonebraker on NoSQL and enterprises". Comm. ACM, Vol 54, No. 8, 2011, pp. 1011

[10] ChristofStrauch "NoSQL Databases" [Lecture Notes] University Hochschule der Medien, Stuttgart, 2014, pp. $17-33$.

[11] "Comparison of various NoSQL databases". [Online] Available: http://kkovacs.eu/cassandra-vs-mongodb-vscouchdb-vs-redis [Accessed Jan 15th, 2015]

[12] Warren, Ian, Andrew Meads, SatishSrirama, ThiranjithWeerasinghe, and Carlos Paniagua. "Push Notification Mechanisms for Pervasive Smartphone Applications." Pervasive Computing, IEEE, Vol 13, No. 2, 2014, pp. 61-71.

[13] Hauswirth, Manfred, and Mehdi Jazayeri. "A component and communication model for push systems." Software Engineering_ESEC/FSE'99. Springer Berlin Heidelberg, Vol. 1687, 1999, pp. 20-38.

[14] "Google Cloud Messaging (GCM)". [Online]. Available: http://developer.android.com/google/gcm/server.html [Accessed Jan 15th, 2015]

[15]"Apple Push Notification Service (APNS)". [Online]. Available:

https://developer.apple.com/library/ios/documentation/ $\mathrm{N}$ etworkingInternet/Conceptual/RemoteNotificationsPG/C hapters/ApplePushService.html [Accessed Jan 15th, 2015]

[16] Fox, Mark A., Peter F. King, SeetharamanRamasubramani, Bruce K. Martin Jr, and Stephen S. Boyle. "Method and apparatus for informing wireless clients about updated information." U.S. Patent 6,654,786, issued November 25, 2003.

[17] Shuang Zhao1,2, Patrick P. C. Lee3, John C. S. Lui3, Xiaohong Guan1, Xiaobo Ma1, Jing Tao1, "CloudBased Push-Styled Mobile Botnets: A Case Study of Exploiting the Cloud to Device Messaging Service", Proceeding ACSAC '12 Proceedings of the 28th Annual Computer Security Applications Conference, 2012, pp. 119-128.

[18] Charland, Andre, and Brian Leroux. "Mobile application development: web vs. native." Communications of the ACM, Vol 54, No. 5, 2011, pp. 49-53.

[19] Henning Heitkötter, Sebastian Hanschke, Tim A. Majchrza, "Comparing cross-platform development approaches for mobile applications", Department of Information Systems, University of Münster, Münster, Germany, 8th International Conference on Web Information Systems and Technologies 2012.

[20] Dalmasso I, Datta S K, Bonnet C, Nikaein N, "Survey, comparison and evaluation of cross platform mobile application development tools." Wireless Communications and Mobile Computing Conference (IWCMC), 2013 9th International. IEEE, 2013.

[21] Ribeiro, André, and Alberto Rodrigues da Silva. "Survey on Cross-Platforms and Languages for Mobile Apps." Quality of Information and Communications Technology (QUATIC), 2012 Eighth International Conference on the IEEE, 2012.

[22] Hartmann, Gustavo, Geoff Stead, and AsiDeGani. "Cross-platform mobile development." Tribal, Lincoln House, The Paddocks, Tech. Rep, 2011.

[23] Yoo, Hee Yong, and Suh Hyun Cheon. "Visualization by information type on mobile device." Proceedings of the 2006 Asia-Pacific Symposium on Information Visualisation-Volume 60. Australian Computer Society, Inc., 2006.

[24] "Marmalade SDK". [Online]. Available: https://www.madewithmarmalade.com/marmalade/howmarmalade-works [Accessed Jan 29th 2015]

[25] Dickson, Jared, "Xamarin Mobile Development", Technical Library, Paper 167, 2013. http://scholarworks.gvsu.edu/cistechlib/167

[26] "Qt Documentation". [Online]. Available: http://doc.qt.io/ [Accessed March 19 $\left.{ }^{\text {th }} 2015\right]$ 